\title{
Simple interventions can greatly improve clinical documentation: a quality improvement project of record keeping on the surgical wards at a district general hospital
}

Peter Glen, Naomi Earl, Felix Gooding, Emily Lucas, Nicole Sangha, Steve Ramcharitar

Great Western Hospital Foundation Trust

\begin{abstract}
Clinical documentation is an integral part of the healthcare professional's job. Good record keeping is essential for patient care, accurate recording of consultations and for effective communication within the multidisciplinary team. Within the surgical department at the Great Western Hospital, Swindon, the case notes were deemed to be bulky and cumbersome, inhibiting effective record keeping, potentially putting patients' at risk. The aim of this quality improvement project was therefore to improve the standard of documentation, the labelling of notes and the overall filing.
\end{abstract}

A baseline audit was firstly undertaken assessing the notes within the busiest surgical ward. A number of variables were assessed, but notably, only $12 \%(4 / 33)$ of the case notes were found to be without loose pages. Furthermore, less than half of the pages with entries written within the last 72 hours contained adequate patient identifiers on them. When assessing these entries further, the designation of the writer was only recorded in one third $(11 / 33)$ of the cases, whilst the printed name of the writer was only recorded in $65 \%(21 / 33)$ of the entries.

This project ran over a 10 month period, using a plan, do study, act methodology. Initial focus was on simple education. Afterwards, single admission folders were introduced, to contain only information required for that admission, in an attempt to streamline the notes and ease the filing. This saw a global improvement across all data subsets, with a sustained improvement of over $80 \%$ compliance seen. An educational poster was also created and displayed in clinical areas, to remind users to label their notes with patient identifying stickers. This saw a 4-fold increase $(16 \%-68 \%)$ in the labelling of notes.

In conclusion, simple, cost effective measures in streamlining medical notes, improves the quality of documentation, facilitates the filing and ultimately improves patient care.

\section{Problem}

At the Great Western Hospital, Swindon, the documentation and record keeping of patient notes on the surgical ward were felt to be substandard when compared to other wards. Case notes were bulky and cumbersome and not ordered chronologically. In addition, patient notes were often hard to locate, impairing the efficiency of the ward round. As a result, entries of ward rounds often contained insufficient clinical data and fell short of basic record keeping recommendations.

\section{Background}

Clinical documentation is an integral part of every doctors job. Good record keeping is essential for patient care, accurate recording of consultations and for effective communication within the multidisciplinary team. Documentation is a legal requirement and is a fundamental factor if a medico-legal issue were to be raised whereby the healthcare professional and the hospital trust could be held accountable. The General Medical Council (GMC) outlines the requirements within Good Medical Practice (2013),[1] stating that one must, "keep clear, accurate, and legible notes, report relevant clinical findings, document decisions made and by whom, document the information given to patients, document any drugs or other treatment prescribed and document who is making the record and when." They go on to state that notes should be kept safely, in accordance with local data protection guidelines avoiding any loose pages.

In addition to this, the Royal College of Physicians England (RCP), have set out criteria that should be adhered to when recording clinical notes.[2] This advises that each page should have at least three unique patient identifiers; and for each entry, the most senior person present, time and date, and the name, designation, and signature of the writer.

Medical records can consist of handwritten entries, emails, clinical letters, laboratory notes, radiographs, photographs, and printouts from medical bedside monitoring equipment. It is, therefore, essential that the many different clinical subsets can be filed appropriately and be easily accessible when required. The records form a permanent account of a patient's illness whilst in hospital, and serve to communicate between different healthcare professionals within the multidisciplinary team.

When a patient seeks legal advice regarding the treatment that they have received, the medical notes will always be reviewed by the 
BMJ Quality Improvement Reports

lawyers to assess for any potential negligence. Furthermore, even if a patient did receive optimal treatment but it had not been documented properly, or the clinical record had been lost, then the healthcare professional could be held accountable.

It stands to reason, that accurately written entries, that are easily legible will help optimise patient safety and facilitate the delivery of excellent patient care. With this in mind, the aims of this project were to improve the:

1. Filing of notes

2. Quality of documentation

3. Labelling of notes with patient identifiers.

\section{Baseline measurement}

Multiple surgical wards exist within the district general hospital. To reduce confounding factors between wards, it was decided to concentrate on the busiest and largest surgical ward, containing 39 bed spaces. The inclusion criteria was simply being an inpatient on the general surgery ward, while patients were excluded if they were absent from the ward with their notes at the time of the audit (for example, if they were in theatre).

The main case notes were assessed for:
1. Were they located in the correct slot within the notes trolley?
2. Whether there were loose pages
3. Were the notes ordered chronologically?

The last 72 hours of note entries were then audited against the RPC guidelines as to having:

1. Three patient identifiers on each page

2. Signature, printed name and designation of the writer

3. The most senior clinician present

4. Time and date.

The baseline audit (figure 1) was carried out in October 2014, consisting of 33 patients out of the available 39 beds. It was clear from the initial baseline data, that there was a global problem with both filing and documentation of the medical records.

Most notably, only $12 \%$ (4/33) of the case notes were found to be without loose pages. Furthermore, less than half of the pages with entries written within the last 72 hours contained adequate patient identifiers on them. When assessing these entries further, the designation of the writer was only recorded in one third (11/33) of the cases, while the printed name of the writer was only recorded in $65 \%(21 / 33)$ of the entries. These were quite alarming results highlighting poor entry recordings and filing of patient notes, directly translating to a reduction in patient safety.

See supplementary file: ds5830.pptx - "Figure 1. Graph showing distribution of data collection of the baseline measurements."

\section{Design}

When considering the underlying problem, it became clear that the overall size of the notes was the main hindrance to effective record keeping. Case notes containing multiple historic entries were greatly increased in overall volume, to the extent that there was not enough room in which to physically file records, and new pages were simply shoved in to the notes loose. This had a knock on effect on the documentation, as the writer would struggle to find the last entry in an attempt to write chronologically, and being behind, would generally rush their entry and thereby omit much necessary data.

After discussion with the healthcare professionals who accessed and used the patient notes, it was felt that the notes were too bulky and needed to be slim lined. Historic notes, although important, were felt not to be generally relevant to the current admission.

In the first instance, an education drive was undertaken. An email was sent to all staff within the surgical department detailing the poor results seen in the baseline data collection, and advising them of the standards and recommendations set out by the GMC and the $\mathrm{RCP}$.

It was then agreed that, in order to minimise the bulk of the notes, single episode folders would be introduced. These folders dramatically streamlined the patient notes by containing only clinical documents from the current admission. The folders could then be easily filed chronologically, with separate sections for medical notes, nursing notes, and results. The folders were then numbered on the spine as per the bed numbers and placed neatly within the trolley, spine up, to save further time. Patient identity stickers were also made readily available at the front of each folder to help facilitate the ease of labelling pages.

Minimal set up costs (approximating £50) were required for this project in the purchase of 40 new ring binder folders, and each colour coded for the section of the ward to which they belonged. A pre-existing secure filing cabinet was then reutilised to be the designated area where the main patient notes would be accessibly stored, should they be needed.

\section{Strategy}

PDSA cycle 1: Following the baseline data collection in October 2014, the single episode patient folders were then introduced to the general surgery ward in November 2014 along with educational emails reminding staff on the requirements of good clinical record keeping. The ward was then re-audited using the same criteria as the baseline data collection. Results were very encouraging (figure 2) with a global improvement of all data subsets, the biggest improvement seen in the proportion of notes without loose pages going up from $12 \%$ to $80 \%$. However, only a mild improvement was seen in the labelling of pages with patient identifiers ( $46 \%$ to $67 \%)$.

PDSA cycle 2: Having seen a generalised improvement in the quality of the documentation, it deemed prudent to explore how the 
notes would then be filed from the folders back into the main notes after the patient was discharged. Twenty sets of case notes were randomly selected from both before and after the introduction of the folders. The notes were assessed for both loose pages and whether they were correctly filed in the corresponding sections: clinical history, letters, investigations, nursing notes, and drug charts. When considering the "before" group, the inclusion criteria was that they had to have been admitted and discharged before November 2014, without readmission. The "after" group had to have been admitted and discharged after the introduction of the single episode folders.

An overall improvement was seen in the general filing of the main patient notes after the introduction of the single episode folders. The average number of sections without loose page pages rose dramatically (52\% to $86 \%$ ), while the filing of the clinical history in the correct place rose from $63 \%$ to $92 \%$. Significant increases were also seen in the filing of nursing notes (44\% to $82 \%$ ) and of drug charts $(44 \%$ to $82 \%)$.

PDSA cycle 3: Having three patient identifiers on each page was also highlighted as an area that could have been improved further. A general trend had been noted during the project that the surgical admissions unit (SAU) proforma was being poorly labelled with patient stickers. Strategies to improve the labelling of the SAU proforma involved repeated educational emails to the department and presenting our data at the surgical consultants' monthly meeting, with the aim that they would encourage their respective teams to better their documentation. In addition, a poster was designed, called "Identi-TRI", and displayed in clinical areas (figure 3 ) to act as a visual aid reminder of the RCP and GMC guidelines. The results of this saw a dramatic four fold increase (16\% to $68 \%)$ in the labelling of the SAU proforma with the required three patient identifiers on each page (figure 4).

PDSA cycle 4: The final cycle involved further education with emails to the surgical staff stressing the importance of good record keeping. A universal improvement from the baseline audit was seen, with furthered improvement in near all areas following the PDSA cycle 1.

See supplementary file: ds5831.pptx - "Data set showing results of PDSA cycle 1 (figure 2), Identi-TRI poster (figure 3 ) and labeling of SAU proforma (figure 4)."

\section{Post-measurement}

Over each PDSA cycle, a general improvement in the record keeping and labelling of notes was seen. The folders facilitated the filing of notes and helped to reduce the number of loose pages considerably. The identi-tri campaign showed a focused improvement increasing the number of pages with three identifiers on each page. An overall, sustained compliance of over $80 \%$ was seen in each area measured (figure 5).

See supplementary file: ds5832.pptx - "Figure 5. Graph showing sustained compliance of over $80 \%$ in all measured areas."

\section{Lessons and limitations}

The main take home message is that simple, low cost strategies and interventions can have a dramatically positive effect. However, despite frequent reminders and educational emails, best practice was not always followed in clinical record keeping. Despite an overall improvement of over $80 \%$ in all areas identified, one in five entries still fell short of the GMC's best practice guidelines. When speaking informally with colleagues to ascertain reasons, the general consensus was that at very busy times, the quality of the documentation would fall short simply due to time pressure.

The focus of the project was also limited to one surgical ward and the surgical teams. Medical record keeping effects all specialities within the hospital, and further work and resources would be required to implement the folders onto other wards.

The surgical admissions proforma was unnecessarily long, consisting of 11 pages and it was deemed rather cumbersome to stick labels onto every page. Future focus could, therefore, be aimed at streamlining the proforma. In addition, proposals for the development of a short online learning module on record keeping as part of a new healthcare professionals induction, are in the early phases.

\section{Conclusion}

Through education, the introduction of single episode patient folders, and through the "identi-tri" campaign it has been shown that the overall standard of medical record keeping has not only improved, but there has been a sustained improvement over the last year. Well organised and easy to find notes will have a positive impact on the quality of the note keeping, but will also facilitate efficient ward rounds, thus freeing up time for other jobs. It is estimated that the interventions detailed could save at least 30 minutes of time per day for each member of the medical team. Over a year this would equate to an estimated saving of $£ 26,097.50$ in hours saved, a considerable sum when considering the minimal set up costs.

\section{References}

1. The General Medical Council. Good Medical Practice. 2013. paras 19-21e.

2. Royal College of Physicians. RCP Approved. Generic Medical Record Keeping Standards. 2009. [ONLINE] Available from:

https://www.rcplondon.ac.uk/sites/default/files/genericmedical-record-keeping-standards-2009.pdf (Accessed May 2015).

\section{Declaration of interests}

Nothing to declare.

\section{Acknowledgements}


Steve Ramcharitar (consultant cardiologist), Badri Chandrasekaran (consultant cardiologist).

\section{Ethical approval}

This project was deemed an improvement study and not a study on human subjects, and local policy meant that ethical approval was not required. 